\title{
Stability of deoxynivalenol and ochratoxin A Through the Bread-Making Process
}

\author{
Vicente Sanchis (I), Arnau Vidal (I), Antonio J Ramos (I), Sonia \\ Marin (I) \\ (I) UdL - University of Lleida- Agrotecnio (Rovira Roure 191; 25198 Lleida (SPAIN))
}

\section{Resumo}

Different studies show the high presence of mycotoxins, especially deoxynivalenol (DON) and ochratoxin A (OTA), in products of high consumption like wheat bakery products. DON has been linked with human gastroenteritis. OTA is a nephrotoxic mycotoxin which has been classified as a possible human carcinogen, in the group $2 \mathrm{~B}$, by the International Agency for Research on Cancer (IARC). Processing of cereals may affect mycotoxins content; however, the extent of DON and OTA reduction during thermal food processing seems to be quite variable and dependent on the processing conditions applied: temperature, time and size of cereal product. The fate these mycotoxins during the breadmaking process was studied. In particular, toxins content was analysed in mixed baking ingredients before kneading, after fermentation and proofing, and finally after baking. Fermentation and proofing were carried out at $30^{\circ} \mathrm{C}$ for $1 \mathrm{~h}$, while baking was performed at different temperature levels (from 170 to $210^{\circ} \mathrm{C}$ ) and baking times from 45 to $135 \mathrm{~min}$, in a full factorial design. Overall, the level of DON increased from unkneaded mix to fermented dough, and decreased due to baking; this trend depended on the initial concentration of DON in the flour. The level in the bread was significantly lower than in the initial mix of ingredients. On the other hand, OTA had a more stable behaviour and the content of OTA in the final bread was the same of the initial flour. To improve the knowledge of DON and OTA reduction during baking, DON and OTA degradation kinetics were studied in cake analogues using different temperature (from 140 to $200^{\circ} \mathrm{C}$ ) and time (from 0 to 40 minutes) levels; small cake analogues with faster heat 
transmission were used. The obtained kinetics showed again the higher stability of OTA than DON through baking. As conclusion, the design of bakery product processes may help to control mycotoxins in final products, because although quite stable, their levels can be reduced to some extent. Mycotoxins have been always considered as stable compounds; however, in depth knowledge of the processing steps that may lead to some reduction (although limited) and those which can stimulate their release from conjugated forms will definitely help in their control in finished foodstuffs.

Palavras-Chave: Deoxynivalenol, Ochratoxin A, Bread-making process Agência de Fomento: 